Ann. Biol. anim. Bioch. Biophys., I970, 10 (I), 5-I3.

\title{
INFLUENGE DE TRAITEMENTS \\ A L'HORMONE SOMATOTROPE BOVINE SUR LA CROISSANCE PONDÉRALE D'AGNEAUX NORMAUX OU HYPOPHYSECTOMISÉS
}

\author{
A. VEZINHET et L. DAUZIER \\ avec la collaboration technique de E, Bouthier et T. Chéry \\ Laboratoive de Recherches de la Chaire de Zootechnie, \\ École nationale supérieure agrononique \\ Centre de Recherches de Montpellier \\ Institut national de la Recherche agronomique
}

\section{RÉSUMÉ}

Après avoir montré que la BGH était sans effet sur le gain de poids d'agneaux normaux traités de la naissance jusqu'à l'âge de roo jours, nous avons étudié l'influence d'un traitement chronique à l'hormone somatotrope bovine chez des agneaux dont la croissance avait été arrêtée par ablation de l'hypophyse.

Dans tous les cas envisagés, le traitement a amélioré de façon très nette le gain de poids vif par rapport à des animaux hypophysectomisés non traités.

Les valeurs des gains de poids obtenues chez les hypophysectomisés traités à la BGH se sont sensiblement rapprochées de celles obtenues pour des animaux ayant subi un simulacre d'opération.

Nous avons envisagé d'autre part l'influence de reliquats hypophysaires sur le gain de poids d'agneaux hypophysectomisés traités ou non à la BGH. Il ressort de cette étude que la présence de reliquat inférieur à $30 \mathrm{p}$. I ooo est sans importance.

Enfin, pour trois doses de BGH utilisées (I, 3 et $5 \mathrm{mg}$ par $\mathrm{kg}$ de poids vif) la plus forte de ces doses semble manifester une certaine toxicité.

\section{INTRODUCTION}

Le contrôle hypophysaire de la croissance a été étudié chez de nombreux mammifères, le degré d'inhibition de la croissance observé après hypophysectomie variant 
avec l'espèce. Chez le Cobaye, de nombreux auteurs, notamment Mrtcheis, et al. (I954), KNOBIL, et GrEeP, (I959) ont montré que l'hypophysectomie n'affecte pas le développement corporel. Chez le Rat (WALKER et al, I952) et le Lapin (VEZINHET, I968 b) l'ablation de l'hypophyse n'entraînerait une inhibition de la croissance qu'à partir d'âges respectivement voisins de 28 et roo jours. Enfin, chez des espèces telles que le Chat (McPhaIr, I935), le Chien (Houssay et Hug, I923); (DANDy et REICHERT, I938), la Souris (LostroH et Li, I958), l'Agneau (VezinheT, I968 a) et le Singe (KNOBIL et Greep, I959) 1'hypophysectomie détermine un arrêt immédiat de la croissance.

On constate également des réponses variables à des traitements chroniques à la $\mathrm{GH}$ selon l'espèce et l'origine de l'hormone. C'est ainsi que la $\mathrm{GH}$ bovine $(\mathrm{BGH})$ et porcine augmentent significativement et durablement le poids et la taille des rats hypophysectomisés (BECKs et al., I949 ; PAPKOFF et LI, I962), la BGH étant également active chez des rats normaux (Evans et al., r948). Par contre, des GH d'Homme et de Singe ont un effet bref sur le développement corporel du Rat hypophysectomisé (Li et al., I959) tandis que des extraits hypophysaires d'Oiseaux sont sans action (Hazer,wood et Hazelwood, I96I). Chez le Singe, la GH de Simiens est active, la BGH n'ayant pas d'effet (KNOBIL, et GREEP, I959). LosTroH et I, (I958) ont montré que la Souris réagit de façon semblable au Rat à des injections de BGH. Enfin, BRUMBY (I959) a observé une action non significative d'un traitement chronique à la $\mathrm{BGH}$ chez la Génisse.

Chez l'Agneau où l'hypophysectomie entraîne un arrêt immédiat et durable de la croissance pondérale (VEzINHET, I968 a), il n'existe aucune donnée relative à l'influence d'un traitement à l'hormone somatotrope. Dans cette étude sont rapportés les résultats obtenus chez des agneaux normaux et hypophysectomisés traités de façon chronique à la BGH.

\section{MATÉRIEL E'T MÉTHODES}

Ce travail a porté sur 53 agneaux Mérinos d'Avles répartis dans différents lots expérimentaux qui figurent au tableau I. La BGH en traitement chronique est administrée par voie intramusculaire, trois fois par semaine, à la dose de $3 \mathrm{mg} / \mathrm{kg}$ de poids vif chaque fois.

En outre, sur 7 agneaux hypophysectomisés à 25 jours nous avons testé l'effet de deux autres doses de BGH : I et $5 \mathrm{mg}$ par $\mathrm{kg}$ de poids vif, administrées jusqu'à Ioo jours.

La survie des animaux privés d'hypophyse a été assurée par un traitement à la cortisone dans les conditions précédemment décrites (VEzINHET, I968 a). Dans tous les cas l'hypophysectomie a été contrôlée histologiquement par l'examen en coupes sériées des selles turciques après coloration au tétrachrome de Herlant.

Lorsque des reliquats hypophysaires ont été observés nous en avons estimé l'importance relative par rapport au volume moyen d'hypophyses d'agneaux du même âge. Nous avons cherché ensuite quelle pourrait être l'influence de ces reliquats sur l'évolution du gain de poids.

Les croissances des animaux hypophysectomisés traités à la BGH ont été comparées d'une part à des agneaux hypophysectomisés et d'autre part à des agneaux témoins ayant subi une opération simulée. 
TABLEAU I

Protocole expérimental

\begin{tabular}{|c|c|c|}
\hline $\begin{array}{l}\text { Désignation } \\
\text { des lots }\end{array}$ & $\begin{array}{c}\text { Lots expérimentaux } \\
\text { (Nbre d'animaux et sexe) }\end{array}$ & Nature du traitement \\
\hline Lot I & $\begin{array}{l}\text { Agneaux intacts } \\
\quad 3 \text { mâles } \\
3 \text { femelles }\end{array}$ & $\begin{array}{l}\text { Traitement à la } \mathrm{BGH} \text { de la naissance à } 100 \text { jours, } \\
\text { à la dose de } 3 \mathrm{mg} \text { par } \mathrm{kg} \text { de poids vif, trois fois } \\
\text { par semaine. }\end{array}$ \\
\hline Lot II & $\begin{array}{l}\text { Agneaux intacts } \\
3 \text { mâles } \\
3 \text { femelles } \\
\end{array}$ & $\begin{array}{l}\text { Solution saline } 9 \text { p. } 1000 \text { de la naissance à } 100 \text { jours }\left({ }^{1}\right) \\
\text { (lot témoin du lot } 1 \text { ). }\end{array}$ \\
\hline Lot III & $\begin{array}{c}\text { Agneaux hypophysectomisés } \\
\text { a } 25 \text { jours } \\
3 \text { mâles } \\
3 \text { femelles } \\
\end{array}$ & $\begin{array}{l}\text { Sans traitement. } \\
\text { Abattage à } 100 \text { jours. }\end{array}$ \\
\hline Lot IV & $\begin{array}{c}\text { Agneaux hypophysectomisés } \\
\text { à } 25 \text { jours } \\
\geq \text { mâles } \\
3 \text { femelles }\end{array}$ & $\begin{array}{l}\text { Traitement à la BGH de } 25 \text { à } 100 \text { jours à la dose de } \\
3 \mathrm{mg} \text { par } \mathrm{kg} \text { de poids vif, trois fois par semaine. }\end{array}$ \\
\hline Lot $\mathrm{V}$ & $\begin{array}{c}\text { Agneaux ayant subi } \\
\text { un simulacre d'opération } \\
\text { à } 25 \text { jours } \\
3 \text { mâles } \\
\geq \geq \text { femelles }\end{array}$ & $\begin{array}{l}\text { Sans traitement, abattage à } 100 \text { jours } \\
\text { (lot témoin des lots III et IV). }\end{array}$ \\
\hline Lot VI & $\begin{array}{l}\text { Agneaux hypophysectomisés } \\
\text { à } 50 \text { jours } \\
: 3 \text { mâles } \\
5 \text { femelles }\end{array}$ & Sans traitement, abattage à 150 jours. \\
\hline Lot VII & $\begin{array}{l}\text { Agneaux hypophysectonisés } \\
\text { à } 50 \text { jours } \\
3 \text { mâles } \\
3 \text { femelles }\end{array}$ & $\begin{array}{l}\text { Traitement à la BGH de } 50 \text { à } 150 \text { jours à la dose } \\
\text { de } 3 \mathrm{mg} \text { par kg de poids vif, trois fois par semaine. }\end{array}$ \\
\hline Lot VIII & $\begin{array}{l}\text { Agneaux ayant subi } \\
\text { un simulacre d'opération } \\
\text { à } 50 \text { jours } \\
2 \text { mâles } \\
\text { ๖ femelles }\end{array}$ & $\begin{array}{l}\text { Sans traitement, abattage à } 150 \text { jours } \\
\text { (lot témoin des lots VI et VII). }\end{array}$ \\
\hline
\end{tabular}

(1) Les volumes de la solution saline injectés, étaient calculés sur la base de ceux utilisés pour la dilution de la BGH.

\section{RÉSULTATS}

Action de la GH sur les animaux normaux

Un traitement chronique à la BGH n'a eu aucun effet significatif sur le gain de poids vif d'agneaux normaux traités de la naissance à un âge de Ioo jours (lot I). 
On a même constaté un gain de poids vif quotidien légèrement mais non significativement inférieur à celui des animaux injectés avec une solution saline à 9 p. I 000 (tabl, 2).

TABLEAU 2

Comparaison des gains quotidiens moyens d'agneaux traités

à l'hormone de croissance bovine pendant les 100 premiers jours de la vie et de témoins injectés avec une solution saline à 9 p. 1000

\begin{tabular}{|c|c|c|c|c|}
\hline & \multicolumn{2}{|c|}{ Mâles } & \multicolumn{2}{|c|}{ Femelles } \\
\hline & Effectif & $\begin{array}{c}\text { Gain moyen } \\
\text { en } g / j\end{array}$ & Effectif & $\begin{array}{l}\text { Gain moyen } \\
\text { en } g / j\end{array}$ \\
\hline Témoins solution saline & $\begin{array}{c}\text { Lot } I I \\
3\end{array}$ & 226 & 3 & 210 \\
\hline Traités BGH. & $\begin{array}{c}\text { Lot } I \\
3\end{array}$ & 207 & 3 & 186 \\
\hline
\end{tabular}

Action de GH sur les animaux hypophysectomisés

En ce qui concerne les animaux hypophysectomisés, l'examen des courbes de croissance (fig. I) permet de constater un effet très net du traitement à l'hormone somatotrope bovine qui ne se manifeste que lorsque les agneaux sont rétablis du choc opératoire, soit environ 20 jours après 1'hypophysectomie en prenant comme critère l'évolution pondérale.

En faisant abstraction de la période postopératoire, les gains de poids moyens quotidiens enregistrés entre 45 et Ioo jours pour les animaux opérés à 25 jours et entre 70 et 50 jours pour les animaux opérés à 50 jours, sont plus élevés pour les animaux traités à la BGH que pour les non traités. Cet effet se manifeste pour les detux sexes (tabl. 3).
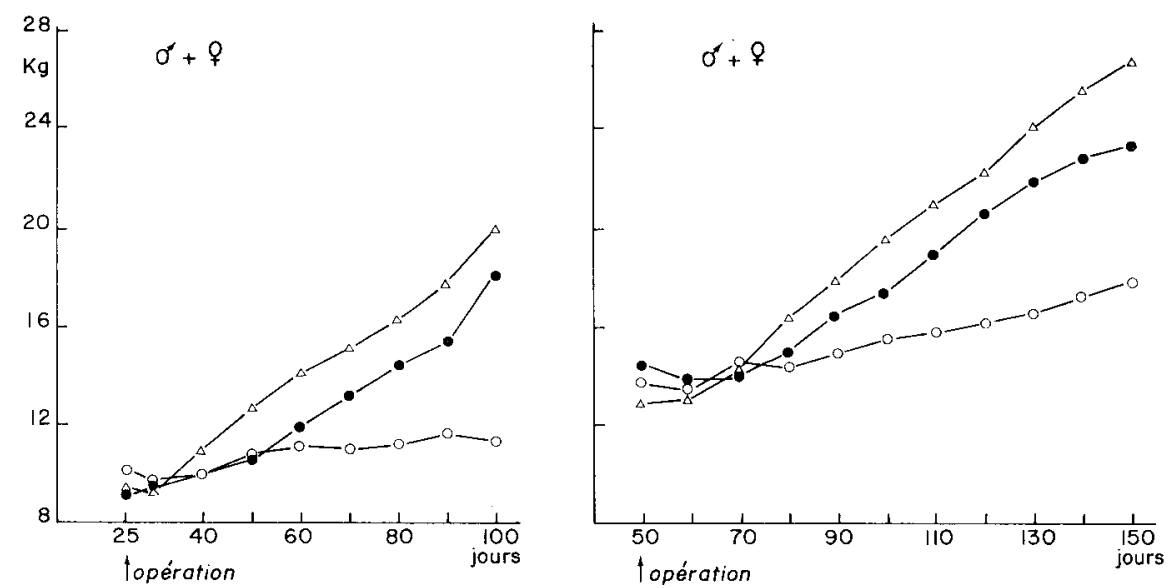

Fíg. I. - Courbes de croissance pondérale d'agneaux hypophysectomisés traités on non à la BGH comparêes à celle d'agneaux ayant subi un simulacre d'opération

-_—— Hypophysectomisés

- ___ Hypophysectomisés traités à la $\mathrm{BGH}$

$\triangle \ldots \ldots$ Simulacre d'opération. 
Cependant, dans tous les cas, sauf un, le gain de poids moyen des agneaux traités est resté inférieur à celui des agneaux témoins.

TABLEAU 3

Comparaison des gains de poids quotidiens moyens d'agneaux hypophysectomisés traités ou non à la $B G H$ et de témoins ayant subi un simulacre d'opération

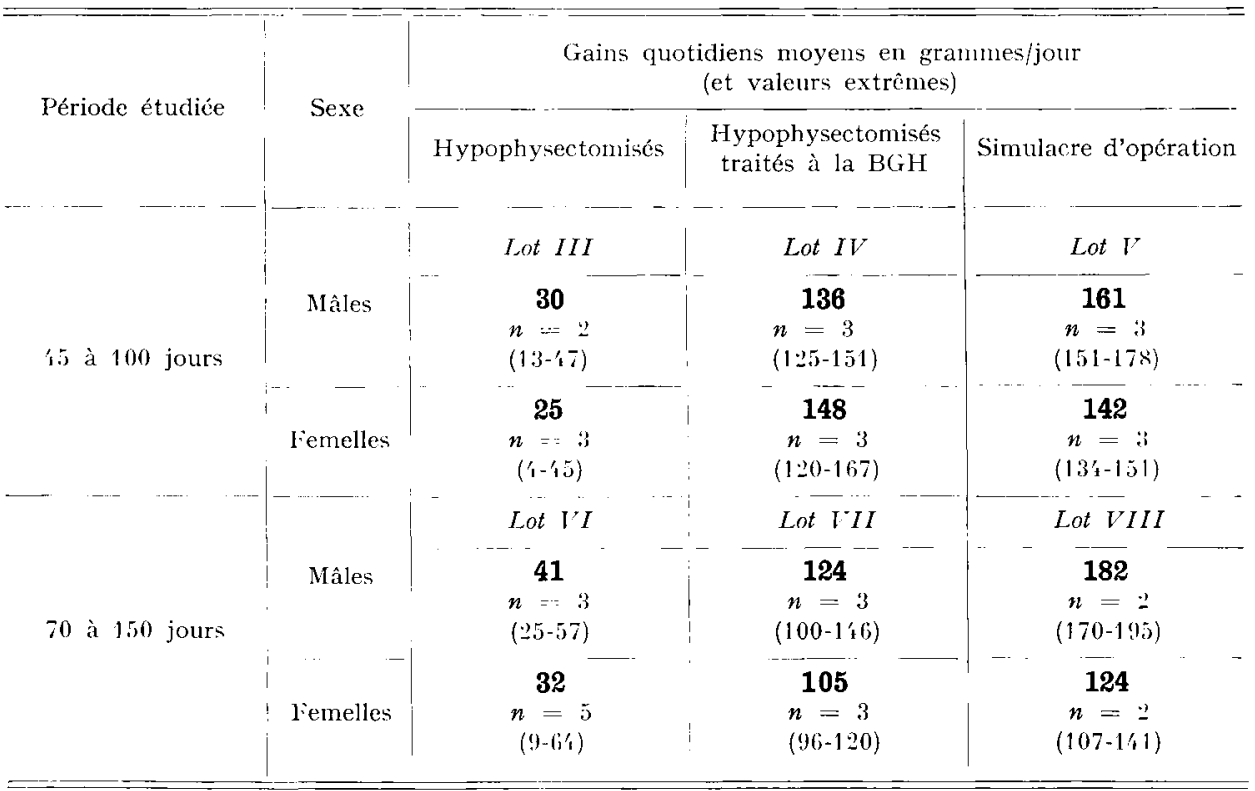

\section{Effet de la dose de $B G H$ injectée}

Pour ce qui est de la dose de BGH injectée, nous avons pu constater que les agneaux hypophysectomisés à 25 jours, traités à la dose de $5 \mathrm{mg}$ par $\mathrm{kg}$ de poids vif, jusqu'à Ioo jours, ont eu entre 45 et Ioo jours un gain de poids inférieur à celui des agneaux traités avec 3 ou I mg (tabl. 4). Il n'y a pas eu, par contre, de différence significative entre les effets produits sur le gain de poids vif, par ces deux dernières doses.

TABLEAU 4

Infuence de la dose de BGH injectée suy le gain de poids quotidien moyen des agneaux hypophysectomisés entre 45 at 100 jours

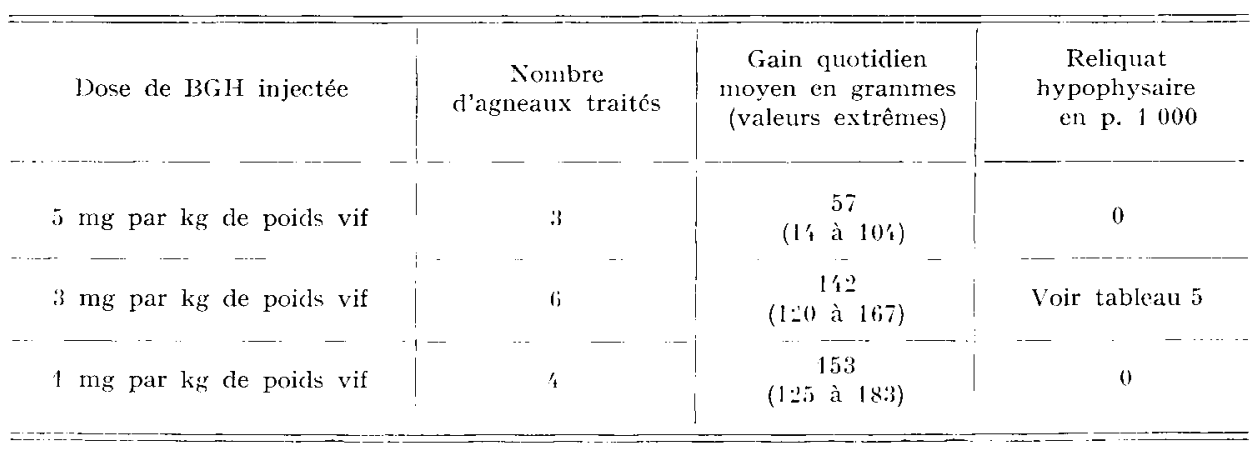




\section{Effet des reliquats hypophysaires}

I1 ressort du tableau 5 que pour des reliquats hypophysaires inférieurs à 30 p. I ooo, il n'y a pas de relation nette entre l'importance du reliquat et le gain de poids vif des animaux hypophysectomisés traités ou non à la $\mathrm{BGH}$, à la dose de $3 \mathrm{mg}$ par $\mathrm{kg}$ de poids vif. Nous n'avons observé aucun reliquat chez les agneaux traités avec des doses de 5 et I mg par $\mathrm{kg}$ de poids vif (tabl. 4). Les reliquats hypophysaires éventuellement observés étaient situés à la périphérie de la selle turcique mais n'ont pas été localisés avec précision par suite de la présence de tissus cicatriciels importants.

\section{TABIEAU 5}

Influence des reliquats hypophysaires sur le gain de poids quotidien moyen d'agneaux hypophysectomisés traités ou non à l'hormone somatotrope

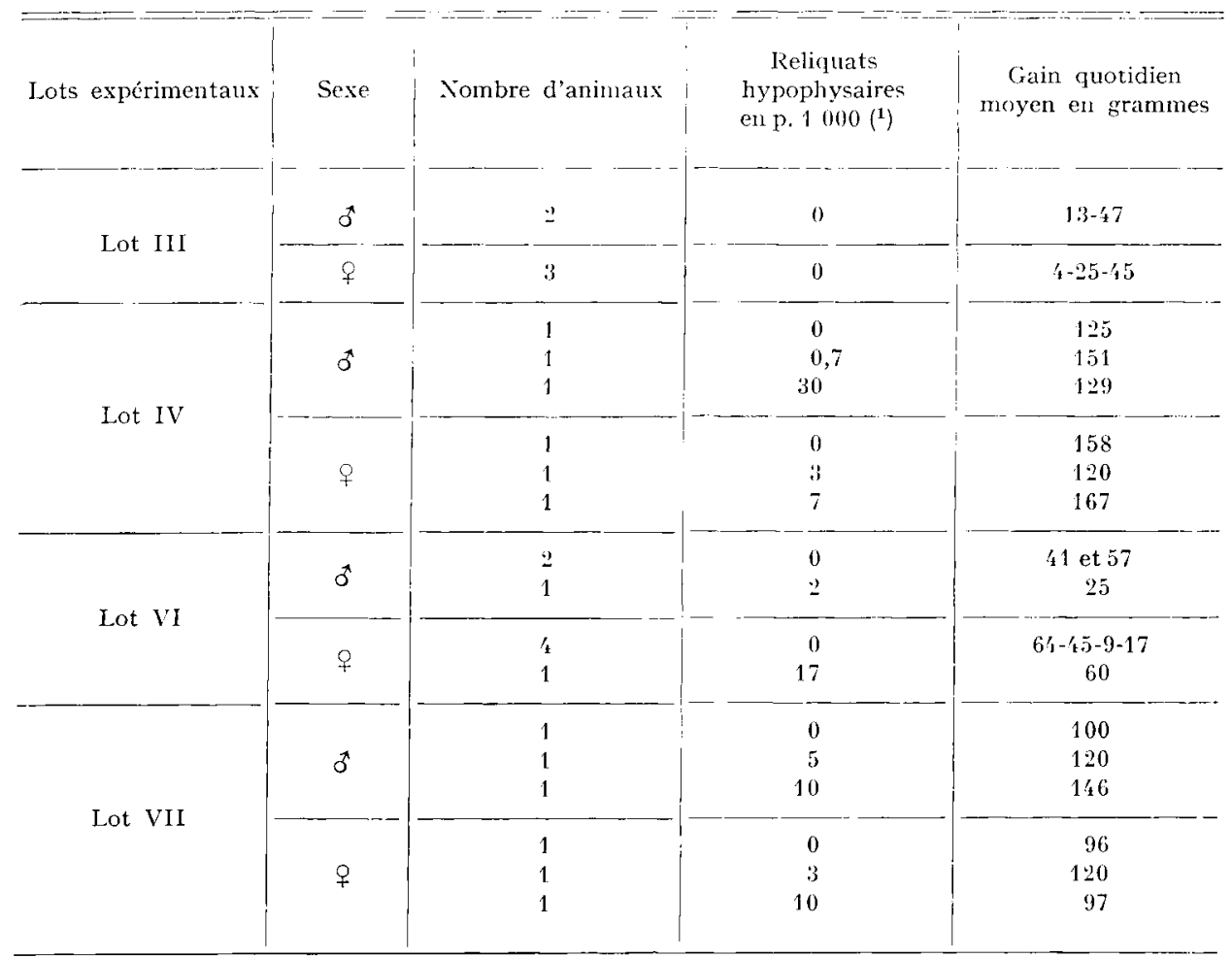

(1) Les volumes des reliquats hypophysaires ont été estimés par rapport à des volumes-témoins d'hypophyses d'animaux intacts du même âge. 


\section{DISCUSSION}

Ces résultats confirment l'importance du contrôle hypophysaire sur la croissance pondérale : 1'hypophysectomie détermine un net ralentissement du développement corporel des agneaux quand l'intervention a lieu dès le $25^{\mathrm{e}}$ jour de la vie ou plus tardivement. L'hormone somatotrope bovine administrée en traitement chronique, à des animaux hypophysectomisés, est capable d'assurer à elle seule un gain de poids vif important quoique légèrement inférieur en moyenne à celui des animaux témoins ayant subi un simulacre d'opération. Dans le cas des agneaux opérés à un âge de 25 jours et traités jusqu'à roo jours, les gains de poids sont identiques à ceux des témoins.

Mais, contrairement à ce qui avait été montré chez le Rat (BECKs et al., I949) 1'hormone de croissance n'a aucun effet sur la croissance pondérale d'agneaux normaux. La spécificité de 1'hormone de croissance ne peut expliquer cette absence d'effet constatée chez les animaux intacts, puisqu'elle s'est révélée active chez les animaux privés d'hypophyse.

En outre, on admet que les $\mathrm{GH}$ bovine et ovine ont des caractéristiques physicochimiques voisines (PAPKoff et L, I, I962).

Des études immunologiques ont montré des similitudes très poussées entre ces deux hormones (HAYASHida et Li, I958, I959; TASHJian et al., I965).

En ce qui concerne les doses de GH que nous avons utilisées, si les doses de I et $3 \mathrm{mg}$ par $\mathrm{kg}$ de poids vif sont efficaces, celle de $5 \mathrm{mg}$ semble manifester une certaine toxicité.

Les travaux réalisés à ce jour ne permettent pas de connaître avec suffisamment de précision le niveau de la sécrétion endogène de $\mathrm{GH}$ chez les Ovins. On note même des différences considérables entre les estimations faites par différents auteurs, notamment WALLACE (I962) et MACHLIN et al. (I968).

Si l'hormone de croissance bovine est capable de déterminer chez les agneaux hypophysectomisés un gain de poids vif proche de celui d'un animal témoin, la composition du croît est différente. C'est ainsi que chez les animaux normaux les dépôts adipeux sont nettement plus importants que chez les animaux hypophysectomisés traités à la BGH (VFZINHET, I967). Si l'on compare les animaux hypophysectomisés traités à ceux non traités, on constate des différences encore plus importantes. L'hypophysectomie détermine une importante surcharge en lipides des carcasses, tandis que la $\mathrm{BGH}$ provoque une mobilisation lipidique qui rend les carcasses d'animaux traités particulièrement maigres.

De plus, la BGH stimule les croissances musculaire et osseuse, tandis que les animaux hypophysectomisés présentent une régression importante de ces deux types de tissus. Ces résultats sont en accord avec de nombreuses observations qui ont révélé que la $\mathrm{GH}$ agissait en favorisant une importante rétention azotée et en stimulant 1'anabolisme protéique (VAN DYKE, I939 ; SzEGo et WhITE, I949 ; RUSSEL, I955 ; KNoBIL et GREE, I959), en même temps qu'elle provoque une mobilisation des lipides de réserve (RABEN et Hol,LENBERG, I958 ; ENGEL et al., I958).

L'étude que nous avons réalisée demande à être complétée par la détermination 
des quantités consommées d'aliments par les différents groupes expérimentaux, ceci dans le but de voir si certaines des différences enregistrées ne peuvent être en partie expliquées par des différences dans la couverture des besoins nutritionnels.

Resu pour publication on septembre 1969.

\section{SUMMARY}

EFFECT OF B. G. H. ON THE, WEIGHT GAIN OF HYIOPHYSECTOMIZED

OR NORMAL I,AMHS

Our investigations have shown that bovine growth hormone was ineffectual on the weight gain of normal lambs treated from o to Ioo days of age. liurther experiments were undertaken to investigate the effect of B.G.H. on hypophysectomized lambs.

In all cases, B.G.H. treatment induced an increase in weight gain as compared to control hypophysectomized lambs. The rate of gain of treated hypophysectomized lambs came very close to that of sham-hypophysectomized control animals.

Weight gain was irrespective of remaining functional pituitary cells provided that the remainders dicl not exced 3 per cont in volume ot the pituitary gland.

The highest of the three 13.G.H. doses administrered ( $, 3,5 \mathrm{mg}$ per $\mathrm{kg}$ live weight) seemed rather poisonous.

\section{RÉFÉRENCES BIBLIOGRAPHIQUES}

Becks H., Astivg G. W., Simpsox M. Ji, Li C. H., Evass H. M., I949. The growth of hypophysectonized female rats following chronic treatment with pure pituitary growth hormone. Grozth, 13, I $75-189$.

l3rumbs P. J., 1959. The influence of growth hormone on growth in young cattle. X. Z. J. agric. Res. 2, $684-690$.

1) ANd W. H., Rachert $1 \%$ L., 1938. Studies on experimental hypophysectomy in dogs. Johns. Hopkins Hosp. Hull., 62, 122-158.

linget, H. R., Halliax I., Siligl S., Bergenstal 1)., 1958. Viffect of growth hormone on plasma unesterified fatty acid and level of hypophysectonized rats. Proc. Soc. cxp. Biol. Med., 98, 753.

Iivaxs H. M., Stmpson M. J., I-I C. H., 1948. The gigantism produced in normal rats by injection of pituitary growth homone. Growth, 12, I5-32.

HAYASHIDA T., I,i C. H., I958. The immunological investigation on bovine pituitary growth hormone. Endocrinology, 63, 487-497.

Hayasinda T., J. C. H., I959. A comparative immunological study of pituitary growth hormone from various species. Endocrinology, 65, 944-956.

1[AzELWOND R. L., HAZELwOOD B. S., r96I. Effects of avian and rat pituitary extracts on tibia growth and blood composition. Proc. Soc. exp. Biol. Med., 108.

Houssiy 13-A., Hug E., I923. Influence des lésions infundibulo-hypothalamique; sur la croissance. C. R. Soc. Biol., 83, $5 \mathrm{I}-53$.

KNoBil E., GRetP R. O., I959. The physiology of growth hormoril " $:$ I': ar references to its action in the Khesus Monkey and the "Species Specificity "problem. Recent Prog. Horm. Res., 15, $I-69$.

Lostron A. J., Li C. H., I958. Effect of growth hormone and thyroxine on body weight of hypophysectomized $\mathrm{C}\left({ }^{3} \mathrm{H}\right)$ mice. Endocrinology, 62, $484-492$.

Li C. H., PAPKOHF H., JoRdan C. W., I959. Difference in biological behaviour between primates aud beef on whole pituitary growth hormone. Proc. Soc. exp. Biol. Med., 100, 44-4.5.

Machlix L. J., Takahasil Y., Mokino N., Ifertelfend F., Gordon R. S., Kipnis I)., I965. In: PEcile A., Mullek E. L. Groteth Hormone, 292, 305. Excerpta Medica liounclation. Amsterdam.

McPril M. K, 1935. Hypophysectomy of the cat. Proc. R. Soc. Ser. B., 117, 45-6z.

Mitciell M. I., Gulliemin R., Selye H., r954. The effect of somatotrophic hormone on the growth of normal an hypophysectomized guinea pigs. Endocrinology, 54, I I I-If. 
PAPKoff H., Li C. H., I962. In : DorfmaN R. I., Methods in hormone research, vol. 2, 67I-704, Academic Press, New York.

Raben M. S., HollenberG C. H., I958. Effect of growth hormone on fatty acids. J. clin. Invest. $37,922$.

Russel J. A., 1955. In : Smith R. W., GaEbler O. H., Hypophyseal grozoth hormone nature and actions, $\mathbf{2 6 1}$, Mc Graw Hill. New York.

Szego C. M., White A., I949. The influence of growth hormone on fasting metabolism. Endocri nology, 44, I $50-166$.

Tashjian A. H., Levine L., Whilhelmi A. E., I965. Immunochemical relatedness of porcine, bovine, ovine and primate pituitary growth hormone. Endocrinology, 7\%, 563-573.

VAN DYke H. B., r939. The physiology and pharmacology of the pituitary body. Vol. II, Univ. Chicago Press. Chicago.

VEzinhet A., I967. The influence of hypophysectomy and growth hormone on growth in the lamb., p. 39. Excepta Medica International Congress, séries $n^{\circ} \mathrm{I}_{42}$, Amsterdam.

Vezinhet A., Ig68 a. Effet de l'hypophysectomie sur la croissance pondérale de l'Agneau. $C$. $R$. Acad, Sci., 266, 388-390.

Vezinhet A., I968 b. Effet de l'hypophysectomie sur la croissance pondérale du Lapin. C. R. Acad. Sci., 266, 2348-235I.

Walker D. G., Asling C. W., Simpson M. E., LI C. H., Evans H. M., I952. Structural alterations in rats hypophysectomized at six days of age and their correction with growth hormone. Anat. Rec., 114, I9-47.

WALlACE A. L. C., I962. Immunological estimation of sheep growth hormone. J. Endocr., 24, I7I-I78. 